\title{
Editorial
}

Digestion

\section{Are Biomarkers Still Helpful in Hepatocellular Carcinoma?}

\author{
Rolf Lamerz \\ Medical Department II, Klinikum Campus Grosshadern, Ludwigs-Maximilians-University, Munich, Germany
}

Since the first description of $a$-fetoprotein (AFP) as the main serum and tissue tumor marker for hepatocellular carcinoma (HCC) in the 1960s and its detection in germ cell tumors, a tremendous number of clinical studies have investigated this biomarker - particularly in patients with benign chronic liver diseases - up to the development of HCC. The limitations that emerged inspired the search for additional markers intended as adjuncts for a combination with AFP in order to improve the utility in the clinical setting. According to an updated broad review from the National Academy of Clinical Biochemistry (NACB) laboratory medicine practice guidelines for the use of tumor markers in different tumor entities including liver cancer [1], only AFP was recommended as the best investigated biomarker in the screening and early detection of patients at a high risk of HCC, for determining prognosis and for the monitoring of treatment. Among numerous other biomarkers, the fucosylated fraction of AFP (AFP-L3), des-gamma-carboxyprothrombin (DCP) or PIVKA II (prothrombin produced by vitamin $\mathrm{K}$ absence or antagonism II, comprehensive review in [2]) and glypican 3 (GPC-3), especially, were judged as upcoming markers needing further investigation. The most critical application in HCC was, and still is, the surveillance of patients at a high risk of HCC and a reaction upon increased suspicion. Former recommendations in the USA and Europe included surveillance by ultrasound in combination with AFP mea-

\section{KARGER}

E-Mail karger@karger.com

www.karger.com/dig surement and, in the case of detection of a suspicious focus of a certain size, additional radiological investigation by CT or MRI and, if HCC was confirmed by imaging or biopsy, a decision to treat. This surveillance-step combination was still maintained at the Asian Oncology Summit 2009 [3], by the WGO guideline from 2010 [4] and was even extended by the Japanese Society of Hepatology, which, for surveillance other than ultrasound, recommended three biomarkers (AFP, AFP-L3 and DCP) every 3-4 months [for hepatitis B virus (HBV)-related or HCVrelated cirrhosis] or every 6 months (for chronic hepatitis $\mathrm{B} / \mathrm{C}$ or cirrhosis of other etiology) $[5,6]$. In the last practice guideline of the American Association for the Study of Liver Diseases (AASLD) from 2010 [7, 8], surveillance of patients at risk for HCC was recommended only 6-monthly by ultrasound, omitting AFP and the algorithm for small nodules changed by $<1 \mathrm{~cm}$ in diameter (3-monthly control) and $>1 \mathrm{~cm}$ (4-phase multidetectorrow $\mathrm{CT}$, dynamic contrast-enhanced MRI or, in the case of an untypical result for HCC, a biopsy). A similar clinical practice HCC guideline was published this year by the European Association for the Study of the Liver, European Organization for Research and Treatment of Cancer (EASL-EORTC) [9]. It is of note that one of the biggest American working groups for medical practice guidelines, the National Comprehensive Cancer Network (NCCN), evaluating most of their recommendations as evidence category $2 \mathrm{~A}$ plus strength of consensus, has (c) 2013 S. Karger AG, Basel

0012-2823/13/0872-0118 $\$ 38.00 / 0$
Prof. Dr. Rolf Lamerz

Medical Department II, Klinikum Campus Grosshadern, Ludwigs-Maximilians-University Marchionini-Strasse 15 DE-81377 München (Germany)

E-Mail rolf.lamerz@med.uni-muenchen.de 
maintained (even in their most recent HCC guideline, version 2.2012) surveillance of patients at risk for HCC by ultrasound and AFP every 6-12 months, and considers a rising AFP level, besides liver mass or nodule, an indication for liver imaging exploration [10]. Recently, a longterm study on HCC in advanced HCV using AFP, DCP and AFP-L3 in patients of the Hepatitis C Antiviral LongTerm Treatment against Cirrhosis (HALT-C) trial disappointed by detecting only mild-to-moderate elevations of all the biomarkers used and elevations more often in those patients with chronic hepatitis and advanced fibrosis than in HCC, thus qualifying the biomarkers as only poor predictors of HCC [11]. In an AFP study in surveillance of compensated cirrhotic patients for small HCC detection, they were deemed similarly ineffective [12]. However, a more recent French AFP study underscores a significant improvement of the performance of the Milan criteria concerning liver transplantation for HCC by the significant prediction of tumor recurrence by AFP [13]. The search for new additional HCC biomarkers is ongoing [14] and has not yet been optimally resolved, although a prospective cohort study on small HCC using serum HCCR1 [15] looks promising, as does another on serum DKK1 [16] and a third on plasma osteopontin [17] in combination with AFP.

The secure detection of HCC in small biopsies $>1 \mathrm{~cm}$ in diameter is also not devoid of problems, in spite of more refined rules by an international working party classification and a final international consensus. In this respect, the additional use of biomarkers has been suggested and is still under investigation $[18,19]$.

In this issue, Ertle et al. [20] present a prospective German single-center cohort study on the conventional biomarkers AFP and DCP, determined by a commercial assay conducted in a cohort of European patients with HCC of different etiology $[\mathrm{n}=164 ; 18 \%$ had HBV, $17 \%$ had HCV, $14 \%$ were alcoholics, $21 \%$ had nonalcoholic steatohepatitis (NASH), 17\% were cryptogenic and $74 \%$ had cirrhosis] and a control cohort of nonmalignant liver diseases ( $n=422 ; 55 \%$ had viral hepatitis, $19 \%$ had cirrhosis, others had NASH, primary sclerosing cholangitis and autoimmune hepatitis). The aim was to investigate the diagnostic contribution of AFP and DCP, via their single or combined use under cut-off optimization, and also their influence by examining clinical factors. Concerning sensitivities for AFP $(\geq 20 \mathrm{ng} / \mathrm{ml})$ or DCP $(\geq 7.5 \mathrm{ng} / \mathrm{ml}) \mathrm{re}$ lated to HCC etiology, combined marker rates (AFP and/ or DCP) in all HCC cases increased from 53\% (HBV) over $74 \%$ (HCV) to $82-84 \%$ (NASH, alcoholic or cryptogenic etiology cases) which appears to underscore the higher

Are Biomarkers Still Helpfull in HCC? detection rate of HCC by biomarkers in nonviral etiology. In the final evaluation at optimized cut-offs for AFP (10 $\mathrm{ng} / \mathrm{ml})$ and DCP $(5 \mathrm{ng} / \mathrm{ml})$, the sensitivity rates for all HCC cases were 55\% (AFP) or 64\% (DCP) at almost 95\% specificity, and in combination reached $78 \%$ at $89 \%$ specificity. For early stage HCC disease [ 72 TNM cases $\leq 2$ and 48 Barcelona Clinic Liver Cancer (BCLC) cases $\leq \mathrm{A}$ ], sensitivity rates of $38-39 \%$ (AFP) and $46-48 \%$ (DCP) at about $95 \%$ specificity and in combination of $58-65 \%$ at a specificity near $90 \%$ were obtained. By ROC analysis, AUC values were similar for AFP (0.88) and DCP (0.87) and higher for their combination (0.91) for all HCC patients. For early-stage disease, AUC values were reduced for AFP (both 0.84) and DCP $(0.81 / 0.79)$ and in combination to $0.87 / 0.80$. This was in contrast to advanced stages (TNM $>2$ and BCLC $>$ A) yielding higher values for AFP $(0.92 / 0.90)$ and DCP $(0.92 / 0.90)$ and in combination 0.95/0.93. In patients with noncirrhotic HCC, AUC for DCP (0.93) significantly exceeded that for AFP (0.84) with no difference evident in cirrhotic HCC patients. Positive predictive values adjusted for their respective positive prevalence showed slightly higher values for DCP than for AFP and for all HCC than for early-stage HCC cases. They were all $>30-40 \%$, however, which was thus significant for attribution of a positive AFP and DCP test for HCC; in contrast, adjusted negative predictive values were below $10-20 \%$, and were thus not relevant for the exclusion of HCC by a negative AFP or DCP test. According to linear regression analysis, besides a correlation of AFP and DCP with liver function (Model for End-Stage Liver Disease and Child-Turcotte-Pugh scores) and staging, liver enzymes ( $\gamma \mathrm{GT}$, AST and ALT) correlated significantly with the biomarkers $(\mathrm{p}<0.0001)$ in both cohorts, in contrast to a significant correlation only for AFP in early-stage disease and no correlation for either marker with gender, age and etiology.

A special item of the study is its focus on the increasing relevance of the risk of HCC in patients with nonalcoholic fatty liver disease (NAFLD) which may progress to HCC even in the absence of apparent cirrhosis; this was already investigated by the authors in a former publication [21]. The menace of HCC in NAFLD in the USA and Western countries has recently been described; it is stimulated by increasing cases of obesity, metabolic syndrome and type 2 diabetes which lead to many alterations and molecular pathway disturbances [22]. Furthermore, an AASLD practice guideline for the diagnosis and management of NAFLD [23] was recently published, citing a worldwide prevalence of NAFLD ranging from 6.3 to $33 \%$ and prevalence of NASH cirrhosis of between 3-5\%. Ac- 
cording to a Japanese review, NAFLD accounts for $1-5 \%$ of all HCC (male-predominant with a median age of 72 years) and for 10-75\% of HCC arising in noncirrhotic livers [24]. In conclusion, the study by Ertle et al. [20] on the serological detection of HCC of varied etiology in European patients by use of a cut-off optimized measurement of AFP and DCP, with the relatively higher detection rate in NASH, alcoholic steatohepatitis, cryptogenic and noncirrhotic etiology cases, offers a valuable combination for HCC detection of viral and increasingly nonviral causes of this disease. Such detection includes early-stage disease which can ultimately result in patients benefitting from a more successful treatment procedure [25].

\section{References}

$\checkmark 1$ Sturgeon CM, Duffy J, Hofmann BR, Lamerz $\mathrm{R}$, Fritsche HA, Gaarenstroom $\mathrm{K}$, et al: National Academy of Clinical Biochemistry Laboratory Medicine Practice Guidelines for use of tumor markers in liver, bladder, cervical, and gastric cancer. Clin Chem 2010;56:e1e48.

-2 Inagaki Y, Tang W, Makuuchi M, Hasegawa K, Sugawara Y, Kokudo N: Clinical and molecular insights into the hepatocellular carcinoma tumour marker des- $\gamma$-carboxyprothrombin. Liver Int 2011;31:22-35.

$\checkmark 3$ Poon D, Anderson BO, Chen LT, Tanaka K, Lau WY, Cutsem EV, et al: Management of hepatocellular carcinoma in Asia: consensus statement from the Asian Oncology Summit 2009. Lancet Oncology 2009;10:111-118.

$\checkmark 4$ Ferenci P, Fried M, Labrescque D, Bruix J, Sherman M, Omata M, et al: Hepatocellular carcinoma (HCC). A global perspective. J Clin Gastroenterol 2010;44:239-245.

5 Izumi N: Diagnostic and treatment algorithm of the Japanese Society of Hepatology: a consensus-based practice guideline. Oncology 2010;78(S1):78-86.

6 Kudo M: Japan's successful model of nationwide hepatocellular carcinoma surveillance highlighting the urgent need for global surveillance (editorial). Liver Cancer 2012;1: 141-143.

7 Bruix J, Sherman M: Management of hepatocellular carcinoma: an update (AASLD practice guideline). http://www.aasld.org/practiceguidelines/Documents/Bookmarked\%20 Practice\%20Guidelines/HCCUpdate2010. pdf

8 Bruix J, Sherman M: Management of hepatocellular carcinoma: an update (AASLD practice guideline). Hepatology 2011;53:10201022 .
$>9$ European Association for the Study of the Liver, European Organization for Research and Treatment of Cancer. EASL-EORTC clinical practice guidelines: management of hepatocellular carcinoma. J Hepatol 2012;56: 908-943.

10 National Comprehensive Cancer Network (NCCN) Guidelines, version 2.2012, assessed 10/28/11 nccn.org. Hepatobiliary carcinoma.

11 Sterling RK, Wright EC, Morgan TR, Seeff LB, Hoefs JC, Di Biscelgie AM, et al: Frequency of elevated hepatocellular carcinoma (HCC) biomarkers in patients with advanced hepatitits C. Am J Gastroenterol 2012;107:64-74.

12 Giannini EG, Marenco S, Borgonova G, Savarino V, Farinati F, Del Poggio P, et al: Alphafetoprotein has no prognostic role in small hepatocellular carcinoma identified during surveillance in compensated cirrhosis. Hepatology 2012:56:1371-79.

13 Duvoux C, Roudot-Thoraval F, Decaens T, Pessione F, Badran H, Piardi T, et al: Liver transplantation for hepatocellular carcinoma: a model including alpha-fetoprotein improves the performance of Milan criteria. Gastroenterol 2012;143:986-994.

14 Bertino G, Ardiri A, Malaguarnera M, Bertino N, Calcagno GS: Hepatocellular carcinoma serum markers. Semin Oncol 2012;39:410433.

15 Jirun P, Zhang G, Ha SA, Kim HK, Yoo J, Kim S, et al: HCCR-1 for detecting small hepatocellular carcinoma latent in a cirrhotic liver: a prospective cohort study. Gut 2012;61:15141515.

16 Shen Q, Fan J, Yang XR, Tan Y, Zhao W, Xu $\mathrm{Y}$, et al: Serum DKK1 as a protein biomarker for the diagnosis of hepatocellular carcinoma: a large-scale, multicentre study. Lancet Oncology 2012;13:817-826.
17 Shan S, Plymoth A, Ge S, Feng Z, Rosen HR, Sangrajrang S, et al: Identification of osteopontin as a novel marker for early hepatocellular carcinoma. Hepatology 2012;55:483490.

18 Lamerz R: Commentary. Improvement of histological biopsy diagnosis of hepatocellular carcinoma by biomarkers? Gut 2011;60: 881-882.

19 Sherman M: Histological diagnosis of early hepatocellular carcinoma (editorial). Hepatology 2011;53:1427-1429.

20 Ertle JM, Heider D, Wichert M, Keller B, Kueper R, Hilgard P, et al: A combination of alpha-fetoprotein and des-gamma-carboxy prothrombin is superior in detection of hepatocellular carcinoma. Digestion 2013;87: 121-131.

21 Ertle J, Dechene A, Sowa JP, Penndorf V, Herzer K, Kaiser G, et al: Non-alcoholic fatty liver disease progresses to hepatocellular carcino$\mathrm{ma}$ in the absence of apparent cirrhosis. Int J Cancer 2011;128:2436-2443.

22 Baffy G, Brunt EM, Caldwell SH: Hepatocellular carcinoma in non-alcoholic fatty liver disease: an emerging menace. J Hepatol 2012; 56:1384-1391.

23 Chalasani N, Younossi Z, Lavine JE, Diehl AM, Brunt EM, Cusi K, et al: The diagnosis and management of non-alcoholic fatty liver disease : practice guideline by the American Association for the Study of Liver Diseases, American College of Gastroenterology, and the American Gastroenterological Association. Am J Gastroenterol 2012;107:811-826.

24 Hashimoto E, Tokushige K: Hepatocellular carcinoma in non-alcoholic steatohepatitis: growing evidence of an epidemic? Hepatol Res 2012;42:1-24.

25 Lin S, Hoffmann K, Schemmer P: Treatment of hepatocellular carcinoma: a systematic review. Liver Cancer 2012;1:144-158. 